\title{
Best One-Sided Approximation of Some Classes of Functions of Several Variables by Haar Polynomials
}

\author{
Alexander N. Shchitov \\ Dnipro, Ukraine \\ an_shchitov@rambler.ru
}

Keywords: Haar system, Haar polynomials, exact constant, exact value, function of several variables, module of continuity, approximation, integral metric, uniform metric.

\begin{abstract}
Exact values of the best one-sided approximation by Haar polynomials have been obtained in the integral and uniform metrics for some classes of the functions of several variables defined using modulus of continuity $\omega(f ; \mathbf{t})$ and $\omega_{\rho_{i}}(f ; \delta)$.
\end{abstract}

\section{Introduction}

The system of Haar functions, orthonormal on [0,1], was introduced in 1909 [1]. The properties of the Haar system and Fourier-Haar coefficients were studied by many scientists, for example in the articles of P.L. Ulyanov [2], Z. Ciesielski [3], B.I. Golubov [4], I.I. Sharapudinov [5], S.S. Volosivets [6], A.N. Shchitov [7, 8] and others. Some of the obtained results in this area can be found in the review article [9].

In approximation theory a lot of works are devoted to the problems of the approximation of functions of one and several variables by polynomials in the Haar system and partial Fourier-Haar sums. We should emphasize the significance of the obtaining in the approximation theory of the exact results for the values of the function approximation.

In the case of one variable in the articles N.P. Khoroshko $[10,11]$ in the uniform and integral metrics were obtained the exact estimates of the approximation errors of the classes of the continuity functions by polynomials in the Haar system and partial Fourier-Haar sums. In the articles N.P. Khoroshko [10], S.B. Vakarchuk and A.N. Schitov [12] were obtained the exact values of the best approximation by polynomials in the Haar system of the functions of boundary variation in the integral metric. In the case of the classes of the differentiable functions of one variable the exact estimates of the approximation errors by polynomials in the Haar system were obtained in the article [13] in the metrics $L_{p}(1 \leqslant p \leqslant$ $\infty)$.

In the case of the functions of several variables in the articles [14]-[17] were obtained the upper bounds of the approximation of the continuity functions by polynomials in the Haar system using the modulus of continuity. P.V. Zaderey and N.N. Zaderey [18], S.B. Vakarchuk and A.N. Shchitov [19] have obtained the exact estimates of the approximation errors for some function classes by polynomials in the Haar system and partial Fourier-Haar sums in the uniform metric. The exact value of the approximation of one function class by the construction of the type "angle" built using the FourierHaar partial sums was obtained in the article [20] in the uniform metric.

Studying of the best one-sided approximation of the functions were started in the articles of G. Freud [21] and T. Ganelius [22]. The problems of one-sided approximation by trigonometric, algebraic polynomials and splines were studied by N.P. Korneichuk, V.G. Doronin, A.A. Ligun, V.F. Babenko and many other scientists. Some of the obtained results in this area can be found in the monograph [23]. In the article [24] was studied the problem of the one-sided approximation by piecewise constant functions of a certain type.

The exact values of the one-sided approximation by polynomials in the Haar system in uniform and integral metrics were obtained by S.B. Vakarchuk and A.N. Shchitov in the article [25] for some classes of the functions of one variable. 
We continue to study the one-sided approximation by Haar polynomials started at the [25]. In the current research we consider the one-sided approximations of the functions of several variables by Haar polynomials in the integral metrics $\varphi(x)$. The exact values will be obtained for some classes of functions defined using modulus of continuity $\omega(f ; \mathbf{t})$ and $\omega_{\rho_{i}}(f ; \delta)$.

\section{Theory}

Let $\mathbb{I}^{d}=\left\{\mathbf{t}=\left(t_{1}, t_{2}, \ldots, t_{d}\right): 0 \leqslant t_{i} \leqslant 1, i=\overline{1, d}\right\}$ is $d$-dimensional cube in the space $\mathbb{R}^{d}$. On the unit segment $\mathbb{I}=[0,1]$ we consider the binary intervals that are defined in the following way: let for an arbitrary number $n_{i}=2^{m_{i}}+k_{i}\left(m_{i} \in \mathbb{Z}_{+}, k_{i}=\overline{1,2^{m_{i}}}\right)$

$$
\delta_{n_{i}} \equiv \delta_{m_{i}}^{k_{i}}=\left(\left(k_{i}-1\right) / 2^{m_{i}}, k_{i} / 2^{m_{i}}\right) \text {. }
$$

On the $[0,1]$ we define the Haar system [1]: $\chi_{1}(t) \equiv \chi_{0}^{(0)}(t) \equiv 1$, and for $n_{i}=2^{m_{i}}+k_{i}\left(m_{i} \in \mathbb{Z}_{+}, k_{i}=\right.$ $\left.\overline{1,2^{m_{i}}}\right)$

$$
\chi_{n_{i}}(t) \equiv \chi_{m_{i}}^{\left(k_{i}\right)}(t)=\left\{\begin{array}{cl}
2^{m_{i} / 2} & , \text { if } t \in \delta_{m_{i}+1}^{2 k_{i}-1}, \\
-2^{m_{i} / 2} & , \text { if } t \in \delta_{m_{i}}^{2 k_{i}}, \\
0 & , \text { if } t \bar{\in} \overline{\delta_{m_{i}}^{k_{i}}},
\end{array}\right.
$$

where $\overline{\delta_{m_{i}}^{k_{i}}}$ is the closure of the set $\delta_{m_{i}}^{k_{i}}$. In the points of discontinuity the Haar functions are equal to the average of the left and right limits of the Haar functions. In the endpoints of the $[0,1]$ the Haar functions are equal to the limit values from the inside of the segment.

Let $\mathbb{N}^{d} \stackrel{\text { df }}{=}\left\{\mathbf{n}=\left(n_{1}, n_{2}, \ldots, n_{d}\right): n_{i} \in \mathbb{N}, i=\overline{1, d}\right\}, \mathbb{N}_{*}^{d} \stackrel{\text { df }}{=}\left\{\mathbf{n}=\left(n_{1}, n_{2}, \ldots, n_{d}\right): n_{i} \in \mathbb{N} \backslash\{1\}, i=\right.$ $\overline{1, d}\}$. The set $\left\{\chi_{\mathbf{n}}(\mathbf{t}) \stackrel{\text { df }}{=} \prod_{i=1}^{d} \chi_{n_{i}}\left(t_{i}\right)\right\}_{\mathbf{n} \in \mathbb{N}^{d}}$ forms the orthonormal system of Haar functions on the $d$ dimensional cube $\mathbb{I}^{d}$. We note that information about Haar system can be found, for example, in the [27]-[30].

Let $C \equiv C\left(\mathbb{I}^{d}\right)$ is the space of functions $f(\mathbf{t})$, which are continuous on the $\mathbb{I}^{d}$, with the norm $\|f\|_{C} \stackrel{\mathrm{df}}{=} \max \left\{|f(\mathbf{t})|: \mathbf{t} \in \mathbb{I}^{d}\right\}$.

For an arbitrary function $f(\mathbf{t})$ from the space $X$ we define the subsets [26]

$$
\begin{aligned}
& \mathbb{P}_{\mathbf{n}}^{+}=\left\{P_{\mathbf{n}}(\mathbf{t}) \in \mathbb{P}_{\mathbf{n}}: P_{\mathbf{n}}(\mathbf{t}) \leqslant f(\mathbf{t}), \mathbf{t} \in \mathbb{I}^{d}\right\}, \\
& \mathbb{P}_{\mathbf{n}}^{-}=\left\{P_{\mathbf{n}}(\mathbf{t}) \in \mathbb{P}_{\mathbf{n}}: P_{\mathbf{n}}(\mathbf{t}) \geqslant f(\mathbf{t}), \mathbf{t} \in \mathbb{I}^{d}\right\},
\end{aligned}
$$

where we mean the inequalities in the sense of "almost everywhere". The value

$$
\begin{gathered}
\widehat{E}_{\mathbf{n}}^{+}(f)_{X} \stackrel{\text { df }}{=} \inf \left\{\left\|f(\cdot)-P_{\mathbf{n}}(\cdot)\right\|_{X}: P_{\mathbf{n}} \in \mathbb{P}_{\mathbf{n}}^{+}\right\} \\
\left(\widehat{E}_{\mathbf{n}}^{-}(f)_{X} \stackrel{\text { df }}{=} \inf \left\{\left\|f(\cdot)-P_{\mathbf{n}}(\cdot)\right\|_{X}: P_{\mathbf{n}} \in \mathbb{P}_{\mathbf{n}}^{-}\right\}\right)
\end{gathered}
$$

is called the best one-sided approximation from below "+" (above "-") of the function $f(\mathbf{t}) \in X$ in the metric of the space $X$ by the subset of the Haar polynomials $\mathbb{P}_{\mathbf{n}}^{+}\left(\mathbb{P}_{\mathbf{n}}^{-}\right)$of the order at more $\mathbf{n} \in \mathbb{N}^{d}$. Taking into account [21] we define also the value

$$
\widetilde{E}_{\mathbf{n}}(f)_{X} \stackrel{\text { df }}{=} \inf \left\{\left\|P_{\mathbf{n}}(\mathbf{t})-p_{\mathbf{n}}(\mathbf{t})\right\|_{X}: P_{\mathbf{n}} \in \mathbb{P}_{\mathbf{n}}^{-}, p_{\mathbf{n}} \in \mathbb{P}_{\mathbf{n}}^{+}\right\} .
$$

For an arbitrary set $\mathcal{M} \subset X$ we define

$$
\widehat{E}_{\mathbf{n}}^{ \pm}(\mathcal{M})_{X} \stackrel{\text { df }}{=} \sup \left\{\widehat{E}_{\mathbf{n}}^{ \pm}(f)_{X}: f \in \mathcal{M}\right\}, \quad \widetilde{E}_{\mathbf{n}}(\mathcal{M})_{X} \stackrel{\text { df }}{=} \sup \left\{\widetilde{E}_{\mathbf{n}}(f)_{X}: f \in \mathcal{M}\right\}
$$


Let $\Phi$ is the set of the the functions $\varphi(x)$ which are even, finite, continuous and nondecreasing on the segment $[0, \infty)$ and match the conditions $\varphi(0)=0 ; \lim _{x \rightarrow \infty} \varphi(x)=\varphi(\infty)=\infty$. If $\varphi(x) \in \Phi$, then we define the set $\varphi(L)$ of all measured on the $\mathbb{I}^{d}$ functions $f(\mathbf{t})$ such that

$$
\|f\|_{\varphi(L)} \stackrel{\mathrm{df}}{=} \int_{\mathbb{I}^{d}} \varphi(f(\mathbf{t})) \mathbf{d t}<\infty,
$$

where dt $\stackrel{\mathrm{df}}{=} \prod_{i=1}^{d} \mathrm{~d} t_{i}$. If $\varphi(x)=|x|^{p}(1 \leqslant p<\infty)$ then $\varphi(L)$ is a norm space $L_{p} \equiv L_{p}\left(\mathbb{I}^{d}\right)$ of the functions which are integrable in the order $p$ and $\|f\|_{\varphi(L)}$ is the norm $\|f\|_{L_{p}}$ in the order $p$, where

$$
\|f\|_{L_{p}} \stackrel{\mathrm{df}}{=}\left\{\int_{\mathbb{I}^{d}}|f(\mathbf{t})|^{p} \mathbf{d t}\right\}^{1 / p} .
$$

It is well known that in the case of the function of one variable $f(t) \in C$ the module of continuity is defined in the following way (see, for example, [31])

$$
\omega(f ; t)=\sup \{|f(x)-f(y)|: x, y \in[0,1],|x-y| \leqslant t\}(t \in[0,1]) .
$$

In the case of the several variables for the function $f(\mathbf{t}) \in C$ we can define several types of the modulus of continuity. By the full module of continuity $f(\mathbf{t}) \in C$ we call the value [32]

$$
\omega(f ; \mathbf{t})=\sup \left\{|f(\mathbf{x})-f(\mathbf{y})|: \mathbf{x}, \mathbf{y} \in \mathbb{I}^{d},\left|x_{i}-y_{i}\right| \leqslant t_{i}, i=\overline{1, d}\right\}\left(\mathbf{t} \in \mathbb{I}^{d}\right) .
$$

We define the module of continuity of the function $f(\mathbf{t}) \in C$ which depends on how is defined the distance $\rho$ between two points $\mathbf{x}$ and $\mathbf{y}$ in the space $\mathbb{R}^{d}$

$$
\omega_{\rho}(f ; \delta)=\sup \left\{|f(\mathbf{x})-f(\mathbf{y})|: \mathbf{x}, \mathbf{y} \in \mathbb{I}^{d}, \rho(\mathbf{x}, \mathbf{y}) \leqslant \delta\right\}\left(0 \leqslant \delta \leqslant \operatorname{diam}_{\rho}\left(\mathbb{I}^{d}\right)\right),
$$

where $\operatorname{diam}_{\rho}(\mathcal{M})$ is the diameter of the set $\mathcal{M}$. Further by the $\rho$ we mean one of the next distances:

$$
\begin{aligned}
\rho_{1}(\mathbf{x}, \mathbf{y}) & =\left\{\sum_{i=1}^{d}\left(x_{i}-y_{i}\right)^{2}\right\}^{1 / 2}, \\
\rho_{2}(\mathbf{x}, \mathbf{y}) & =\sum_{i=1}^{d}\left|x_{i}-y_{i}\right| \\
\rho_{3}(\mathbf{x}, \mathbf{y}) & =\max _{1 \leqslant i \leqslant d}\left|x_{i}-y_{i}\right| .
\end{aligned}
$$

We introduce the next classes of the functions

$$
\begin{gathered}
H_{\omega} \stackrel{\text { df }}{=}\left\{f(\mathbf{x}) \in C: \omega(f ; \mathbf{t}) \leqslant \omega(\mathbf{t}) \forall \mathbf{t} \in \mathbb{I}^{d}\right\}, \\
H_{\omega, \rho_{i}} \stackrel{\text { df }}{=}\left\{f(\mathbf{t}) \in C: \omega_{\rho_{i}}(f ; \delta) \leqslant \omega(\delta) \forall \delta \in\left[0, \operatorname{diam}_{\rho_{i}}\left(\mathbb{I}^{d}\right)\right]\right\}(i=1,2,3),
\end{gathered}
$$

where $\omega(\mathbf{t})\left(\mathbf{t} \in \mathbb{I}^{d}\right)$ and $\omega(\delta)\left(0 \leqslant \delta \leqslant \operatorname{diam}_{\rho_{i}}\left(\mathbb{I}^{d}\right)\right)$ are some given modulus of continuity.

Let us also define:

- $\mathbb{J}^{d}$ is the set of the points $\mathbf{j} \stackrel{\mathrm{df}}{=}\left(j_{1}, \ldots, j_{d}\right)$, where $j_{s}(s=\overline{1, d})$ can takes one of the two values 1 or 2 ;

- $A_{j_{s}} \stackrel{\mathrm{df}}{=}\left\{2 k_{s}\right.$, if $j_{s}=1 ; 2^{m_{s}}-k_{s}$, if $\left.j_{s}=2\right\}(s=\overline{1, d})$;

- $\mathbb{B}_{\mathbf{j h}}^{d} \stackrel{\text { df }}{=}\left\{\mathbf{t} \in \mathbb{I}^{d}: 0 \leqslant t_{s} \leqslant j_{s} h_{s}, s=\overline{1, d}\right\}$, where $\mathbf{j} \in \mathbb{J}^{d}$; 
- $A_{\mathbf{j}}^{d} \stackrel{\mathrm{df}}{=} \prod_{s=1}^{d} A_{j_{s}} ;$

- $A_{\mathbf{j}}^{d} \mathbf{j h} \stackrel{\mathrm{df}}{=} \prod_{s=1}^{d} A_{j_{s}}^{s} j_{s} h_{s}$

- $\mathbf{j h} \stackrel{\mathrm{df}}{=}\left(j_{1} h_{1}, \ldots, j_{d} h_{d}\right)$, where $h_{s} \stackrel{\mathrm{df}}{=} 2^{-\left(m_{s}+1\right)}(s=\overline{1, d})$.

\section{Results}

Lets $\Theta$ is the null element of the space $\mathbb{R}^{d}$.

Theorem 1. Let a function $\varphi(x) \in \Phi, \omega(\mathbf{t})\left(\mathbf{t} \in \mathbb{I}^{d}\right)$ and $\omega(\delta)\left(0 \leqslant \delta \leqslant \operatorname{diam}_{\rho}\left(\mathbb{I}^{d}\right)\right)$ are the arbitrary modulus of continuity. Then for all $\mathbf{n} \in \mathbb{N}_{*}^{d}$ the equalities hold

$$
\begin{gathered}
\widehat{E}_{\mathbf{n}}^{ \pm}\left(H_{\omega}\right)_{\varphi(L)}=\sum_{\mathbf{j} \in \mathbb{J}^{d}} A_{\mathbf{j}}^{d} \int_{\mathbb{B}_{\mathbf{j h}}^{d}} \varphi(\omega(\mathbf{t})) \mathbf{d t}, \\
\widehat{E}_{\mathbf{n}}^{ \pm}\left(H_{\omega, \rho_{i}}\right)_{\varphi(L)}=\sum_{\mathbf{j} \in J^{d}} A_{\mathbf{j}}^{d} \int_{\mathbb{B}_{\mathbf{j h}}^{d}} \varphi\left(\omega\left(\rho_{i}(\mathbf{t}, \Theta)\right)\right) \mathbf{d t} \quad(i=1,2,3) .
\end{gathered}
$$

Proof. To avoid a cumbersome proof of this theorem we consider the case of the functions of two variables without loss of generality. An arbitrary Haar polynomial of the order at most $\mathbf{n}\left(n_{i}=\right.$ $\left.2^{m_{i}}+k_{i}, m_{i} \in \mathbb{Z}_{+}, k_{i}=\overline{1,2^{m_{i}}} ; i=1,2\right)$ matches on the $\mathbb{I}^{2}$ some stepwise function which is constant on the next sets

$$
\begin{gathered}
\triangle_{m_{1}+1, m_{2}+1}^{\nu_{1}, \nu_{2}}, \triangle_{m_{1}+1, m_{2}}^{\nu_{1}, \eta_{2}}, \triangle_{m_{1}, m_{2}+1}^{\eta_{1}, \nu_{2}}, \triangle_{m_{1}, m_{2}}^{\eta_{1}, \eta_{2}} \\
\left(1 \leqslant \nu_{i} \leqslant 2 k_{i} ; k_{i}<\eta_{i} \leqslant 2^{m_{i}} ; i=1,2\right),
\end{gathered}
$$

where $\triangle \triangle_{m_{1}, m_{2}}^{k_{1}, k_{2}} \stackrel{\text { df }}{=} \delta_{m_{1}}^{k_{1}} \times \delta_{m_{2}}^{k_{2}}$.

Let us put for an arbitrary function $f(\mathbf{t}) \in C$

$$
\mathcal{R}_{m_{1}, m_{2}}^{\nu_{1}, \nu_{2}}(f ; \mathbf{t}) \stackrel{\mathrm{df}}{=} f(\mathbf{t})-\sup \left\{P_{\mathbf{n}}(\mathbf{t}): P_{\mathbf{n}}(\mathbf{t}) \leqslant f(\mathbf{t})\right\} \quad\left(\mathbf{t} \in \triangle_{m_{1}, m_{2}}^{\nu_{1}, \nu_{2}}\right) .
$$

Then we can write

$$
\begin{gathered}
\widehat{E}_{\mathbf{n}}^{+}(f)_{\varphi(L)}=\sum_{\nu_{1}=1}^{2 k_{1}} \sum_{\nu_{2}=1}^{2 k_{2}} \int_{\triangle_{m_{1}+1, m_{2}+1}^{\nu_{1}, \nu_{2}}} \varphi\left(\mathcal{R}_{m_{1}+1, m_{2}+1}^{\nu_{1}, \nu_{2}}(f ; \mathbf{t})\right) \mathbf{d t} \\
+\sum_{\nu_{1}=1}^{2 k_{1}} \sum_{\eta_{2}=k_{2}+1}^{2^{m_{2}}} \int_{\triangle_{m_{1}+1, m_{2}}^{\nu_{1}, \eta_{2}}} \varphi\left(\mathcal{R}_{m_{1}+1, m_{2}}^{\nu_{1}, \eta_{2}}(f ; \mathbf{t})\right) \mathbf{d t}+\sum_{\eta_{1}=k_{1}+1}^{2^{m_{1}}} \sum_{\nu_{2}=1}^{2 k_{2}} \int_{\triangle_{m_{1}, m_{2}+1}^{\eta_{1}, \nu_{2}}} \varphi\left(\mathcal{R}_{m_{1}, m_{2}+1}^{\eta_{1}, \nu_{2}}(f ; \mathbf{t})\right) \mathbf{d t} \\
+\sum_{\eta_{1}=k_{1}+1}^{2^{m_{1}}} \sum_{\eta_{2}=k_{2}+1}^{2^{m_{2}}} \int_{\triangle_{m_{1}, m_{2}}^{\eta_{1}, \eta_{2}}} \varphi\left(\mathcal{R}_{m_{1}, m_{2}}^{\eta_{1}, \eta_{2}}(f ; \mathbf{t})\right) \mathbf{d t} .
\end{gathered}
$$

We fix the set $\overline{\triangle_{m_{1}+1, m_{2}+1}^{\nu_{1}, \nu_{2}}}$ and on it's bound we define the function $\mathcal{R}_{m_{1}+1, m_{2}+1}^{\nu_{1}, \nu_{2}}(f ; \mathbf{t})$ by the continuity. By the Weierstrass theorem it is can be found a point $\mathcal{A}_{\nu_{1}, \nu_{2}} \stackrel{\mathrm{df}}{=}\left(a_{\nu_{1}}^{(1)}, a_{\nu_{2}}^{(2)}\right) \in \overline{\triangle_{m_{1}+1, m_{2}+1}^{\nu_{1}, \nu_{2}}}$ such that

$$
\mathcal{R}_{m_{1}+1, m_{2}+1}^{\nu_{1}, \nu_{2}}\left(f ; \mathcal{A}_{\nu_{1}, \nu_{2}}\right)=0 \text {. }
$$

Then we can write

$$
\mathcal{R}_{m_{1}+1, m_{2}+1}^{\nu_{1}, \nu_{2}}(f ; \mathbf{t})=f(\mathbf{t})-f\left(\mathcal{A}_{\nu_{1}, \nu_{2}}\right)
$$


We divide further the integration set $\triangle_{m_{1}+1, m_{2}+1}^{\nu_{1} \nu_{2}}$

on four subsets:

- $\left(\left(\nu_{1}-1\right) h_{1}, a_{\nu_{1}}^{(1)}\right) \times\left(\left(\nu_{2}-1\right) h_{2}, a_{\nu_{2}}^{(2)}\right)$,

- $\left(\left(\nu_{1}-1\right) h_{1}, a_{\nu_{1}}^{(1)}\right) \times\left(a_{\nu_{2}}^{(2)}, \nu_{2} h_{2}\right)$,

- $\left(a_{\nu_{1}}^{(1)}, \nu_{1} h_{1}\right) \times\left(\left(\nu_{2}-1\right) h_{2}, a_{\nu_{2}}^{(2)}\right)$,

- $\left(a_{\nu_{1}}^{(1)}, \nu_{1} h_{1}\right) \times\left(a_{\nu_{2}}^{(2)}, \nu_{2} h_{2}\right)$,

where $\left(b_{1}, b_{2}\right) \times\left(\gamma_{1}, \gamma_{2}\right)$ is the Cartesian product of the segments $\left(b_{1}, b_{2}\right)$ of the coordinate axis $0 t_{1}$ and $\left(\gamma_{1}, \gamma_{2}\right)$ of the coordinate axis $0 t_{2}$. Using (8) after the series of simple calculations we get for an arbitrary function $f(\mathbf{t}) \in H_{\omega}$

$$
\begin{aligned}
\int_{\delta_{m_{1}+1}^{\nu}} \int_{\delta_{m_{2}+1}^{\nu_{2}}} \varphi\left(\mathcal{R}_{m_{1}+1, m_{2}+1}^{\nu_{1}, \nu_{2}}\left(f ; t_{1}, t_{2}\right)\right) \mathrm{d} t_{1} \mathrm{~d} t_{2} \\
\leqslant \int_{0}^{a_{\nu_{1}}^{(1)}-\left(\nu_{1}-1\right) h_{1}} \int_{0}^{a_{\nu_{2}}^{(2)}-\left(\nu_{2}-1\right) h_{2}} \varphi\left(\omega\left(t_{1}, t_{2}\right)\right) \mathrm{d} t_{1} \mathrm{~d} t_{2}+\int_{0}^{a_{\nu_{1}}^{(1)}-\left(\nu_{1}-1\right) h_{1}} \int_{0}^{\nu_{2} h_{2}-a_{\nu_{2}}^{(2)}} \varphi\left(\omega\left(t_{1}, t_{2}\right)\right) \mathrm{d} t_{1} \mathrm{~d} t_{2} \\
\quad+\int_{0}^{\nu_{1} h_{1}-a_{\nu_{1}}^{(1)}} \int_{\nu_{2} h_{2}-a_{\nu_{2}}^{(2)}}^{(1)} \int_{0}^{(2)} \varphi\left(\omega\left(t_{1}, t_{2}\right)\right) \mathrm{d} t_{1} \mathrm{~d} t_{2}+\int_{0}^{\left(\nu_{\nu_{2}}-\left(\nu_{2}-1\right) h_{2}\right.} \varphi\left(\omega\left(t_{1}, t_{2}\right)\right) \mathrm{d} t_{1} \mathrm{~d} t_{2} .
\end{aligned}
$$

Since the function $\varphi(x)$ doesn't decrease on the $[0, \infty)$ and the module of continuity $\omega(\mathbf{t})$ doesn't decrease for each variable then the composition $\varphi(\omega(\mathbf{t}))$ is a nondecreasing function for each variable. From the (9) we have

$$
\begin{gathered}
\iint_{\triangle_{m_{1}+1, m_{2}+1}^{\nu_{1}}} \varphi\left(\mathcal{R}_{m_{1}+1, m_{2}+1}^{\nu_{1}, \nu_{2}}\left(f ; t_{1}, t_{2}\right)\right) \mathrm{d} t_{1} \mathrm{~d} t_{2} \\
\leqslant \int_{0}^{a_{\nu_{1}}^{(1)}-\left(\nu_{1}-1\right) h_{1}} \int_{0}^{h_{2}} \varphi\left(\omega\left(t_{1}, t_{2}\right)\right) \mathrm{d} t_{1} \mathrm{~d} t_{2}+\int_{0}^{\nu_{1} h_{1}-a_{\nu_{1}}^{(1)}} \int_{0}^{h_{2}} \varphi\left(\omega\left(t_{1}, t_{2}\right)\right) \mathrm{d} t_{1} \mathrm{~d} t_{2} \leqslant \\
\leqslant \int_{0}^{h_{1}} \int_{0}^{h_{2}} \varphi\left(\omega\left(t_{1}, t_{2}\right)\right) \mathrm{d} t_{1} \mathrm{~d} t_{2} .
\end{gathered}
$$

Similar to the (8)-(10) we can get from the (7) the next inequality

$$
\begin{aligned}
& \widehat{E}_{\mathbf{n}}^{+}(f)_{\varphi(L)} \leqslant 4 k_{1} k_{2} \int_{0}^{h_{1}} \int_{0}^{h_{2}} \varphi\left(\omega\left(t_{1}, t_{2}\right)\right) \mathrm{d} t_{1} \mathrm{~d} t_{2} \\
&+2 k_{1}\left(2^{m_{2}}-k_{2}\right) \int_{0}^{h_{1}} \int_{0}^{2 h_{2}} \varphi\left(\omega\left(t_{1}, t_{2}\right)\right) \mathrm{d} t_{1} \mathrm{~d} t_{2}+\left(2^{m_{1}}-k_{1}\right) 2 k_{2} \int_{0}^{2 h_{1}} \int_{0}^{h_{2}} \varphi\left(\omega\left(t_{1}, t_{2}\right)\right) \mathrm{d} t_{1} \mathrm{~d} t_{2} \\
&+\left(2^{m_{1}}-k_{1}\right)\left(2^{m_{2}}-k_{2}\right) \int_{0}^{2 h_{1}} \int_{0}^{2 h_{2}} \varphi\left(\omega\left(t_{1}, t_{2}\right)\right) \mathrm{d} t_{1} \mathrm{~d} t_{2}
\end{aligned}
$$


for an arbitrary function $f(\mathbf{t}) \in H_{\omega}$ and an arbitrary $\mathbf{n} \in \mathbb{N}_{*}^{2}$. From (11) we have the upper bound

$$
\widehat{E}_{\mathbf{n}}^{+}\left(H_{\omega}\right)_{\varphi(L)} \leqslant \sum_{\mathbf{j} \in \mathbb{J}^{2}} A_{\mathbf{j}}^{2} \int_{\mathbb{B}_{\mathbf{j h}}^{2}} \varphi(\omega(\mathbf{t})) \mathbf{d t} .
$$

Similar to the (6)-(12) we get the next relations

$$
\widehat{E}_{\mathbf{n}}^{+}\left(H_{\omega, \rho_{i}}\right)_{\varphi(L)} \leqslant \sum_{\mathbf{j} \in \mathbb{J}^{2}} A_{\mathbf{j}}^{2} \int_{\mathbb{B}_{\mathbf{j h}}^{2}} \varphi\left(\omega\left(\rho_{i}(\mathbf{t}, \Theta)\right)\right) \mathbf{d t}(i=1,2,3) .
$$

Let us obtain the lower bound of the value $\widehat{E}_{\mathbf{n}}^{+}\left(H_{\omega}\right)_{\varphi(L)}$. We define the function

$$
G_{0}\left(a, b ; c, d ; t_{1}, t_{2}\right) \stackrel{\mathrm{df}}{=} \begin{cases}\omega\left(t_{1}-a, t_{2}-c\right), & a \leqslant t_{1} \leqslant(a+b) / 2, c \leqslant t_{2} \leqslant(c+d) / 2, \\ \omega\left(b-t_{1}, t_{2}-c\right), & (a+b) / 2 \leqslant t_{1} \leqslant b, c \leqslant t_{2} \leqslant(c+d) / 2, \\ \omega\left(t_{1}-a, d-t_{2}\right), & a \leqslant t_{1} \leqslant(a+b) / 2,(c+d) / 2 \leqslant t_{2} \leqslant d, \\ \omega\left(b-t_{1}, d-t_{2}\right), & (a+b) / 2 \leqslant t_{1} \leqslant b,(c+d) / 2 \leqslant t_{2} \leqslant d .\end{cases}
$$

Firstly, we consider the case of even numbers $n_{i}(i=1,2)$. For an arbitrary module of continuity $\omega(f ; \mathbf{t})$ we define a function $G(\mathbf{t}) \in H_{\omega}$ in the following way

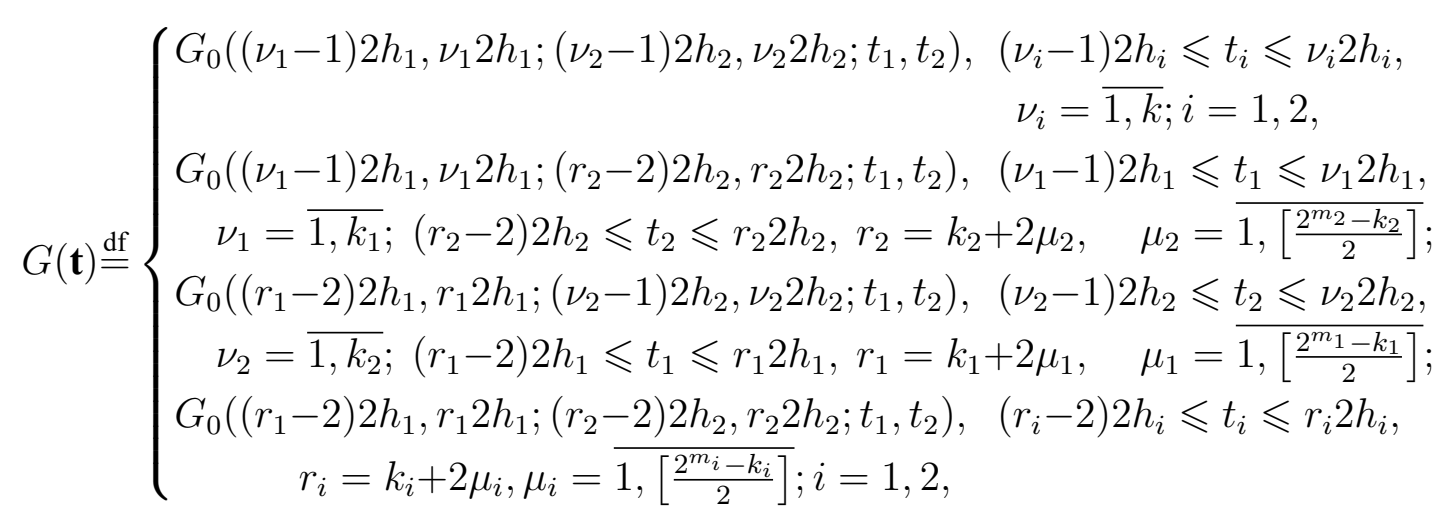

where $[a]$ is the integral part of the number $a \in \mathbb{R}$. It is easy to show that the function $G_{*}(\mathbf{t}) \equiv 0$ everywhere on $\mathbb{I}^{2}$ meets an inequality $G_{*}(\mathbf{t}) \leqslant G(\mathbf{t})$. So due to (15) the function $G_{*}(\mathbf{t})$ is the polynomial of the order $\mathbf{n}$ of the best one-sided approximation from below for the function $G(\mathbf{t})$. After simple calculations in the case of even $n_{i}(i=1,2)$ we obtain the lower bound

$$
\widehat{E}_{\mathbf{n}}^{+}\left(H_{\omega}\right)_{\varphi(L)} \geqslant \widehat{E}_{\mathbf{n}}^{+}(G)_{\varphi(L)}=\int_{\mathbb{I}^{2}} \varphi(G(\mathbf{t})) \mathbf{d t}=\sum_{\mathbf{j} \in \mathbb{J}^{2}} A_{\mathbf{j}}^{2} \int_{\mathbb{B}_{\mathbf{j h}}^{2}} \varphi\left(\omega\left(\rho_{i}(\mathbf{t}, \Theta)\right)\right) \mathbf{d t} .
$$

In the case of odd $n_{i}(i=1,2)$ we should complete the definition of the function $G(\mathbf{t})$ in the following way: if $k_{1}$ and $k_{2}$ are odd and $1-2 h \leqslant t_{i} \leqslant 1(i=\overline{1,2})$ then $G(\mathbf{t})=\omega\left(t_{1}+2 h-1, t_{2}+\right.$ $2 h-1)$, where $h \stackrel{\mathrm{df}}{=} 2^{-(m+1)}$.

It is easy to show the fairness of the inequality (16) in this case. The relation (3) for the value $\widehat{E}_{\mathbf{n}}^{+}\left(H_{\omega}\right)_{\varphi(L)}$ follows from the (12) and (16).

Taking into account (14)-(15) and mentioned above it is not difficult to define the functions which are the extremal functions on the classes $H_{\omega, \rho_{i}}(i=1,2,3)$. Then we can obtain the lower bounds

$$
\widehat{E}_{\mathbf{n}}^{+}\left(H_{\omega, \rho_{i}}\right)_{\varphi(L)} \geqslant \sum_{\mathbf{j} \in \mathbb{J}^{2}} A_{\mathbf{j}}^{2} \int_{\mathbb{B}_{\mathbf{j} \mathbf{h}}^{2}} \varphi\left(\omega\left(\rho_{i}(\mathbf{t}, \Theta)\right)\right) \mathbf{d t} .
$$


From the last relation and (13) we have the equality (4) in the case of the best one-sided approximation from below $\widehat{E}_{\mathbf{n}}^{+}\left(H_{\omega, \rho_{i}}\right)_{\varphi(L)}$.

Similarly we can proof the theorem 1 in the cases of the values $\widehat{E}_{\mathbf{n}}^{-}\left(H_{\omega}\right)_{\varphi(L)}$ and $\widehat{E}_{\mathbf{n}}^{-}\left(H_{\omega, \rho_{i}}\right)_{\varphi(L)}$. The theorem 1 is proved.

Theorem 2. For the given function $\varphi(x) \in \Phi$, the arbitrary modulus of continuity $\omega(\mathbf{t})\left(\mathbf{t} \in \mathbb{I}^{d}\right)$ and $\omega(\delta)\left(0 \leqslant \delta \leqslant \operatorname{diam}_{\rho}\left(\mathbb{I}^{d}\right)\right)$ the equalities hold

$$
\begin{gathered}
\widetilde{E}_{\mathbf{n}}\left(H_{\omega}\right)_{\varphi(L)}=\sum_{\mathbf{j} \in \mathbb{J}^{d}} A_{\mathbf{j}}^{d} \mathbf{j h} \varphi(\omega(\mathbf{j h})) \\
\widetilde{E}_{\mathbf{n}}\left(H_{\omega, \rho_{i}}\right)_{\varphi(L)}=\sum_{\mathbf{j} \in \mathbb{J}^{d}} A_{\mathbf{j}}^{d} \mathbf{j h} \varphi\left(\omega\left(\rho_{i}(\mathbf{j h}, \Theta)\right)\right)(i=1,2,3)
\end{gathered}
$$

for all $\mathbf{n} \in \mathbb{N}_{*}^{d}$.

Proof. Without loss of generality we consider the case of the functions of two variables to proof the theorem 2. On the fixed set $\overline{\triangle_{m_{1}+1, m_{2}+1}^{\nu_{1}, \nu_{2}}}$ for some function $f(\mathbf{t}) \in C$ we define a function

$$
\mathcal{Z}_{m_{1}+1, m_{2}+1}^{\nu_{1}, \nu_{2}}(f ; \mathbf{t}) \stackrel{\text { df }}{=} \inf \left\{P_{\mathbf{n}}(\mathbf{t}): P_{\mathbf{n}}(\mathbf{t}) \geqslant f(\mathbf{t})\right\}-f(\mathbf{t}) \quad\left(\mathbf{t} \in \triangle_{m_{1}+1, m_{2}+1}^{\nu_{1}, \nu_{2}}\right) .
$$

Also we define the function $\mathcal{Z}_{m_{1}+1, m_{2}+1}^{\nu_{1}, \nu_{2}}(f ; \mathbf{t})$ on the bound of the set $\triangle_{m_{1}+1, m_{2}+1}^{\nu_{1}, \nu_{2}}$ by the continuity. By the Weierstrass theorem it is exist a point $B_{\nu_{1}, \nu_{2}}=\left(b_{\nu_{1}}^{(1)}, b_{\nu_{2}}^{(2)}\right) \in{\overline{\triangle_{m_{1}+1, m_{2}+1}^{\nu_{1}, \nu_{2}}}}_{\text {Auch that }}$

$$
\mathcal{Z}_{m_{1}+1, m_{2}+1}^{\nu_{1}, \nu_{2}}\left(f ; B_{\nu_{1}, \nu_{2}}\right)=0 \text {. }
$$

Then we can write

$$
\begin{aligned}
& \int_{\triangle_{m_{1}+1, m_{2}+1}^{\nu_{1}, \nu_{2}}} \varphi\left(\mathcal{Z}_{m_{1}+1, m_{2}+1}^{\nu_{1}, \nu_{2}}(f ; \mathbf{t})+\mathcal{R}_{m_{1}+1, m_{2}+1}^{\nu_{1}, \nu_{2}}(f ; \mathbf{t})\right) \mathbf{d t} \\
& \quad=\left|\triangle_{m_{1}+1, m_{2}+1}^{\nu_{1}, \nu_{2}}\right| \varphi\left(f\left(B_{\nu_{1}, \nu_{2}}\right)-f\left(\mathcal{A}_{\nu_{1}, \nu_{2}}\right)\right)
\end{aligned}
$$

where $\mathcal{R}_{m_{1}+1, m_{2}+1}^{\nu_{1} \nu_{2}}(f ; \mathbf{t})$ is defined by the formula (6) and $f\left(\mathcal{A}_{\nu_{1}, \nu_{2}}\right)$ is defined by the (8). Using the considerations similarly to the mentioned above for the function $f(\mathbf{t}) \in H_{\omega}$ on other sets of the form (5), the properties of the Haar polynomials and based on the (6), (19)-(20) we have

$$
\begin{aligned}
& \widetilde{E}_{\mathbf{n}}(f)_{\varphi(L)} \leqslant 4 h_{1} h_{2}\left\{k_{1} k_{2} \varphi\left(\omega\left(h_{1}, h_{2}\right)\right)+k_{1}\left(2^{m_{2}}-k_{2}\right) \varphi\left(\omega\left(h_{1}, 2 h_{2}\right)\right)\right. \\
& \left.+\left(2^{m_{1}}-k_{1}\right) k_{2} \varphi\left(\omega\left(2 h_{1}, h_{2}\right)\right)+\left(2^{m_{1}}-k_{1}\right)\left(2^{m_{2}}-k_{2}\right) \varphi\left(\omega\left(2 h_{1}, 2 h_{2}\right)\right)\right\} .
\end{aligned}
$$

We have the upper bound from the (21)

$$
\widetilde{E}_{\mathbf{n}}\left(H_{\omega}\right)_{\varphi(L)} \leqslant \sum_{\mathbf{j} \in \mathbb{J}^{2}} A_{\mathbf{j}}^{2} \mathbf{j h} \varphi(\omega(\mathbf{j h})) .
$$

Similarly to the obtaining of the relations (21)-(22) and based on the (6)-(8), (19)-(20) we obtain

$$
\widetilde{E}_{\mathbf{n}}\left(H_{\omega, \rho_{i}}\right)_{\varphi(L)} \leqslant \sum_{\mathbf{j} \in \mathbb{J}^{2}} A_{\mathbf{j}}^{2} \mathbf{j h} \varphi\left(\omega\left(\rho_{i}(\mathbf{j} \mathbf{h}, \Theta)\right)\right) \quad(i=1,2,3) .
$$

The lower bounds can be easy obtained using the functions $G$ which are defined at the proof of the theorem 1 . Thus the theorem 2 is proved.

$$
\begin{aligned}
& \text { Lets } \frac{\mathbf{1}}{\mathbf{n}^{\prime}} \stackrel{\text { df }}{=}\left(\frac{1}{n_{1}^{\prime}}, \cdots, \frac{1}{n_{d}^{\prime}}\right), \text { where } \\
& \qquad n_{i}^{\prime} \stackrel{\text { df }}{=} \begin{cases}2^{m_{i}} & , \text { if } n_{i}=2^{m_{i}}+k_{i}\left(m_{i} \in \mathbb{N} ; k_{i}=\overline{1,2^{m_{i}}-1}\right), \\
2^{m_{i}+1} & , \text { if } n_{i}=2^{m_{i}+1}\left(m_{i} \in \mathbb{Z}_{+}\right) ; i=\overline{1, d} .\end{cases}
\end{aligned}
$$

From the theorems 1-2 it is follows the next theorem. 
Theorem 3. For the arbitrary modulus of continuity $\omega(\mathbf{t})\left(\mathbf{t} \in \mathbb{I}^{d}\right), \omega(\delta)\left(0 \leqslant \delta \leqslant \operatorname{diam}_{\rho}\left(\mathbb{I}^{d}\right)\right)$ and for all $\mathbf{n} \in \mathbb{N}_{*}^{d}$ the equalities hold

$$
\begin{gathered}
\widehat{E}_{\mathbf{n}}^{ \pm}\left(H_{\omega}\right)_{C}=\widetilde{E}_{\mathbf{n}}\left(H_{\omega}\right)_{C}=\omega\left(\frac{\mathbf{1}}{\mathbf{n}^{\prime}}\right) \\
\widehat{E}_{\mathbf{n}}^{ \pm}\left(H_{\omega, \rho_{i}}\right)_{C}=\widetilde{E}_{\mathbf{n}}\left(H_{\omega, \rho_{i}}\right)_{C}=\omega\left(\rho_{i}\left(\frac{\mathbf{1}}{\mathbf{n}^{\prime}}, \Theta\right)\right)(i=1,2,3) .
\end{gathered}
$$

\section{Conclusions}

Exact values of the best one-sided approximation by Haar polynomials have been obtained in the integral $\varphi(x)$ and uniform metrics for classes $H_{\omega}$ and $H_{\omega, \rho_{i}}$ of the functions of several variables. If $d=1$ then from the theorems 1-2 we get the results obtained by S.B. Vakarchuk and A.N. Shchitov in the paper [25].

\section{References}

[1] A. Haar, Zur Theorie der orthogonalen Funktionensysteme, Inaugural-dissertation, GeorgAugust-Universitat, Göttingen, 1909.

[2] P.L. Ulyanov, Series in Haar system, Matem. sbornik. 63(1) (1964) 356-391. (in Russian)

[3] Z. Ciesielski, On Haar functions and on the Schauder Basis of the Space $C_{\langle 0,1\rangle}$, Bulletin de L'academie Polonaise Des Sciences. Serie des sci. math., astr. et phys. 7(4) (1959) 227-232.

[4] B.I. Golubov, On Fourier series of continuous functions with respect to a Haar system, Izv. Akad. Nauk SSSR. Ser. Mat. 28 (1964) 1271-1296. (in Russian)

[5] I.I. Sharapudinov, Approximation of functions in variable-exponent Lebesgue and Sobolev spaces by finite Fourier-Haar series, Sb. Math. 205(2) (2014) 291-306.

[6] S.S. Volosivets, Approximation of functions of bounded p-variation by means of polynomials of the Haar and Walsh systems, Mathematical Notes. 53(6) (1993) 569-575.

[7] A.N. Shchitov, Exact estimates of the Fourier-Haar coefficients of some classes of functions of several variables, Zb. Pr. Inst. Mat. NAN Ukr. 1(1) (2004) 413-427. (in Russian)

[8] A.N. Shchitov, The exact estimates of Fourier-Haar coefficients of functions of bounded variation, International Journal of Advanced Research in Mathematics. 4 (2016) 14-22.

[9] B.I. Golubov, Series with respect to the Haar system, J. Soviet Math. 1(6) (1973) 704-726.

[10] N.P. Khoroshko, On the best approximation in the metric of $L$ to certain classes of functions by Haar-system polynomials, Mathematical notes of the Academy of Sciences of the USSR. 6(1) (1969) 487-491.

[11] N.P. Khoroshko, Uniform approximation in classes of continuous functions by polynomials in the Haar system, Ukrainian Math. J. 22(5) (1971) 611-618. (in Russian)

[12] S.B. Vakarchuk, A.N. Shchitov, On the best approximation of functions of bounded p-variation by Haar polynomials, Bulletin of the Dnipropetrovsk University. Mathematics. 11 (2004) 28-34. (in Russian)

[13] S.B. Vakarchuk, A.N. Shchitov, Estimates for the error of approximation of functions in $L_{p}^{1}$ by polynomials and partial sums of series in the Haar and Faber-Schauder systems, Izvestiya Mathematics. 79(2) (2015) 257-287. 
[14] A.R. Abdulgamidov, On some properties of the Fourier-Haar series of the functions of two variables, Proceedings of the postraduate papers of the Kazan state university: Mathematics, Mechanics, Physics. (1968) 5-33. (in Russian)

[15] N.D. Rishchenko, On the best approximation of the function of the several variables by the step functions, Proccedings of the Dnipropetrovsk state university. (1969) 56-59. (in Russian)

[16] M.N. Ochirov, On approximation of functions of two variables by Fourier-Haar partial sums, Proccedings of the Kazan state university: Function analyse and function theory. 8 (1971) 142145. (in Russian)

[17] L.G. Khomutenko, Uniform approximation of functions of two variables by polynomials in the Haar system, Proccedings of the Dnipropetrovsk state university. (1973) 85-87. (in Russian)

[18] P.V. Zaderey, N.N. Zaderey, Uniform approximations by polynomials in the Haar system on classes of continuous functions of several variables, Zb. Pr. Inst. Mat. NAN USSR. (1984) 6066. (in Russian)

[19] S.B. Vakarchuk, A.N. Shchitov, Uniform approximation of some classes of functions of several variables by polynomials constructed by Haar system and partial sums of the Fourier-Haar series, Zb. Pr. Inst. Mat. NAN Ukr. 1(1) (2004) 42-59. (in Russian)

[20] A.N. Shchitov, On approximation of the continuous functions of two variables by the FourierHaar polynomials, International Journal of Advanced Research in Mathematics. 5 (2016) 23-31.

[21] G. Freud, Uber cinseitige Approximation durch Polynome, I, Acta Sci. Math. (Szeged). 16(2) (1955) 12-28.

[22] T. Ganelius, On the sided approximation by trigonometric polynomials, Math. Scand. 4 (1956) 247-258.

[23] N.P. Korneichuk, A.A. Ligun, V.G. Doronin, Approximation with constraints, Naukova dumka, Kiev, Ukrainian SSR, 1982. (in Russian)

[24] V.G. Doronin, A.A. Ligun, On the study of the best one-sided approximation of some function classes of the continuous functions, Proccedings of the Dnipropetrovsk state university. (1974) 42-49. (in Russian)

[25] S.B. Vakarchuk, A.N. Shchitov, Estimates of the best one-sided approximation of some classes of functions by polynomials constructed on Haar system, Zb. Pr. Inst. Mat. NAN Ukr. 4 (2007) 7-22. (in Russian)

[26] N.P. Korneichuk, Exact constants in approximation theory, Encyclopedia of Mathematics and its Applications, 38, Cambridge University Press, Cambridge, 1991.

[27] G. Alexits, Convergence problems of orthogonal series, Akademia Kiado, Budapest, Hungary, 1961.

[28] B.S. Kashin, A.A. Saakyan, Orthogonal series, Transl. Math. Monogr., 75, Amer. Math. Soc., Providence, RI, 1989.

[29] S.S. Kaczmarz, H. Steinhaus, Theorie der Orthogonalreihen, Monografje Matematyczne, VI, Chelsea Publishing Company, New York, 1951.

[30] I.M. Sobol, Multidimensional quadrature formulas and functions Haar, Nauka, Moscow, USSR, 1969. (in Russian)

[31] N.P. Korneichuk, Extremal problems in the theory of approximation, Nauka, Moskow, USSR, 1976. (in Russian)

[32] N.P. Korneichuk, Splines in approximation theory, Nauka, Moskow, USSR, 1984. (in Russian) 